\title{
The Implications of the Use of Composite Materials in Electromagnetic Device Topology and Shape Optimization
}

\author{
Dong-Hun Kim ${ }^{1}$, Jan K. Sykulski ${ }^{2}$, and David A. Lowther ${ }^{3}$ \\ ${ }^{1}$ School of Electrical Engineering and Computer Science, Kyungpook National University, Daegu 702-701, Korea \\ ${ }^{2}$ School of Engineering and Computer Science, University of Southampton, Southampton SO17 1BJ, U.K. \\ ${ }^{3}$ Electrical and Computer Engineering Department, McGill University, Montreal, QC H3A 2A7, Canada
}

\begin{abstract}
The use of soft magnetic composites (SMCs) provides electromagnetic device designers with serious challenges. While such materials have the potential of allowing novel 3-D topologies to be used, as well as providing major advantages in recycling at the end of the lifetime of the device, little or no design experience exists in this area. This paper proposes the use of sensitivity based topology optimization as a methodology for assisting designers in this task.
\end{abstract}

Index Terms-Electrical machines, shape optimization, soft magnetic composites (SMC), topology optimization.

\section{INTRODUCTION}

$\mathbf{T}$ HE development of new materials, particularly powdered magnetic steels, and improved permanent magnets has the potential of changing the design of electrical machines. These materials are likely to become more common in the foreseeable future as designers are required to consider the entire life cycle of a machine from manufacture to recycling.

Composite materials offer a range of properties which will have a considerable impact on electromagnetic device design. For example, most electrical machines up to now have been pseudo-two-dimensional in their structure, i.e., they have been constructed from laminated materials which, naturally, force a translational structure on the permeable components. The need for laminations is imposed by the requirement to reduce eddy currents which may be induced when the material is subjected to time-varying magnetic fields. Composite materials, constructed from insulated permeable particles, naturally restrict the eddy currents in all directions and this removes the translational constraint. Moreover, manufacturing processes for a device are nearer to those for injection moulded structures than traditional laminated core assemblies.

The major drawback of such materials is seen to be their (relatively) low permeabilities when compared to conventional magnetic steels, which results in more material being needed to carry the required fluxes in the device. This problem might be counterbalanced by making use of truly 3-D structures; however, little experience exists in creating these.

The goal of this paper is to describe the application of a sensitivity-based topology design process for these devices

\section{Sensitivity-BAsed Topology Design}

To deal efficiently with topological optimization of magnetostatic problems in three dimensions, a new algorithm for the sensitivity-based topology design is introduced.

Manuscript received October 07, 2008. Current version published February 19, 2009. Corresponding author: D. A. Lowther (e-mail: david.lowther@mcgill. ca).

Color versions of one or more of the figures in this paper are available online at http://ieeexplore.ieee.org.

Digital Object Identifier 10.1109/TMAG.2009.2012661

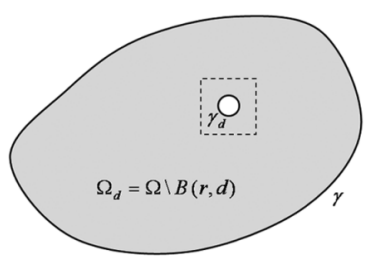

(a)

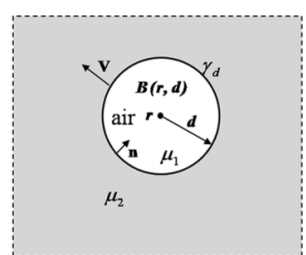

(b)
Fig. 1. Design domain after cutting a small hole. (a) A domain $\Omega \backslash B(r, d)$. (b) An enlarged hole.

\section{A. Definition of the Topological Gradient}

The topological gradient (TG) $G(r)$ gives information on the opportunity to create a small hole $B(r, d)$ with radius $d$, centered at $r$, belonging to the design domain $\Omega$ (as in Fig. 1)

$$
G(r)=\lim _{d \rightarrow 0} \frac{\Psi_{\mathrm{obj}}(\Omega \backslash B(r, d))-\Psi_{\mathrm{obj}}(\Omega)}{\delta(\Omega)}
$$

where $\Psi_{\mathrm{obj}}$ is the objective function, $\Omega \backslash B(r . d)$ is the domain $\Omega$ with the hole $B(r, d)$ and $\delta(\Omega)$ is the area difference of the domain after and before the small hole is present [1], [2]. As seen in (1), the definition of TG is quite different from that of the material sensitivity used commonly in the electromagnetic topology optimization, where the derivative of the objective function with respect to the infinitesimal change of material properties is sought out [3]-[5].

\section{B. Link With the Classical Shape Optimization}

In order to establish a link between the TG and the classical shape sensitivity, let us first compute the derivative of a scalar function $J$ expressed in terms of $\Psi_{\mathrm{obj}}$ and $d$

$$
J(d)=\Psi_{\mathrm{obj}}(\Omega \backslash B(r, d)) .
$$

To achieve this, we consider a small perturbation Fig. 1 which keeps the outer boundary $\gamma$ of the domain invariant (design velocity $\mathbf{V}=0$ on $\gamma$ ) but increases the radius $d$ of the hole $\left(\mathbf{V}=-\mathbf{n}\right.$ on $\left.\gamma_{d}\right)$. In this case, the classical shape sensitivity of (2), i.e., $J^{\prime}$, can be expressed in terms of the magnetic fields 
while the adjoint fields may be based on the shape gradient information presented in [6]

$$
\begin{aligned}
J^{\prime}(d) & =-\int_{\gamma_{d}} L\left(\mathbf{A}_{1}, \boldsymbol{\lambda}_{2}\right) d \gamma \\
L\left(\mathbf{A}_{1}, \boldsymbol{\lambda}_{2}\right) & =\left(\nu_{1}-\nu_{2}\right) \nabla \times \mathbf{A}_{1} \cdot \nabla \times \boldsymbol{\lambda}_{2}
\end{aligned}
$$

where $\mathbf{A}$ is the magnetic vector potential, $\lambda$ the adjoint variable, $v$ the magnetic reluctivity and the subscripts, 1 and 2 , denote the corresponding regions $B(r, d)$ and $\Omega_{d}$, respectively. The adjoint variable may be easily obtained after the adjoint system, a counterpart to the primary system, is constructed and solved - as described in [6] - by adopting the continuum design sensitivity analysis (CDSA) approach. Using a local expansion of $\mathbf{A}$ and $\lambda$ distributed along the circumference, $\gamma_{d}[1],(3)$ is approximated at the center of the hole as

$$
J^{\prime}(d)=-4 \pi d L\left(\mathbf{A}_{1}(r), \boldsymbol{\lambda}_{2}(r)\right)+o(d) .
$$

Finally, the difference in the objective function after and before cutting the hole is obtained as

$$
J(d)-J(0)=\int_{0}^{d} J^{\prime}(\rho) d \rho=-2 \pi d^{2} L\left(\mathbf{A}_{1}(r), \boldsymbol{\lambda}_{2}(r)\right)+o\left(d^{2}\right) .
$$

Thus, comparing (5) with (1), it is deduced that the TG is given by $G(r)=2 L\left(\mathbf{A}_{1}(r), \lambda_{2}(r)\right)$, where the natural extension of the shape gradient is $L\left(\mathbf{A}_{1}(r), \lambda_{2}(r)\right)$. Hence, the topological and the shape gradients differ by a factor 2 .

\section{Optimization Algorithm}

In this paper, the ON/OFF method based on TG values is applied in order to obtain an actual topology in three dimensions within an acceptable computing time. Assuming a volume constraint imposed on the optimization problem, the proposed algorithm involves the following steps:

1) define an objective function and a volume constraint;

2) divide the initial domain into $l$ layers, each consisting of $n$ design cells, and set the iteration number $k$ to be 0 so that each cell is filled with a material;

3 ) solve the primary and adjoint systems;

4) compute the topological gradient $G_{k}(r)$ at the middle points of the cells in each layer, where the TG value is calculated on a plane including all the points;

5) sort the cells by their respective value of TG; the lowest cells are then removed using a certain volume ratio that is typically taken as being between $3 \%$ and $5 \%$ of the material volume at each iteration;

6) check the volume constraint and go to Step 2) $(k \leftarrow k+1)$ if unsatisfactory.

In practice, the proposed method needs only a few iterations as it does not require intermediate material states, nor any penalty functions for a material or an objective function used.

\section{Material CURVES}

Fig. 2 shows typical magnetization curves for laminated steel and for a soft material composite [7].

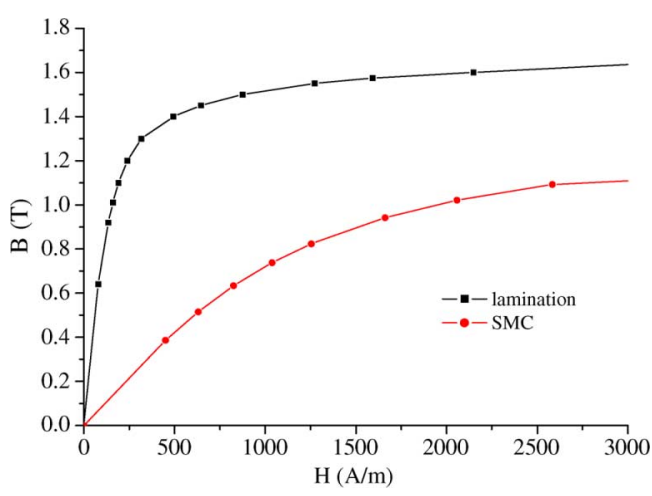

Fig. 2. Magnetization curves of a laminated material and an SMC.

As may be seen, the initial permeability of the composite is much lower than that of a conventional magnetic steel and this means that more material is going to be needed to carry the same flux. At the "knee" point in the laminated material, the $m m f$ needed is about $400 \mathrm{~A} / \mathrm{m}$, whereas at this point the flux density in the composite is only about $25 \%$ of that in laminated steel. However, as the $m m f$ increases and the laminated material is pushed into saturation, this ratio changes rapidly. This would suggest that, to achieve the same performance, a composite based electrical machine may need significantly higher currents or larger windings as well as extra material. Similar findings have been reported before and it is often argued that SMC materials are relatively more suited for machines with low demand in terms of permeability, for example permanent magnet, dc, and synchronous machines, whereas it is more difficult to achieve good results if high permeability is demanded, say in induction, reluctance and stepping motors. However, the purpose of this paper is not to provide a comparative analysis of performance of SMC machines against laminated-core equivalents, but to discuss the special features of the design process based on topology and shape optimization when composite materials are used.

\section{Test PRoblem}

Comparing the performance of two possible designs, one made from a conventional laminated material and the other using SMC, is difficult and depends on the comparison criteria specified. The problem chosen for this example was the spider (or pole piece) of the stator in an exterior rotor BLDC as shown in Fig. 3.

A crucial issue in this type of machine is that of cogging torque and so it was decided to make this the target of the optimal design. Additionally, many of these machines have a restricted size and so a constraint was added to the system such that the material volume of the laminated and composite stators should be the same. In hindsight, this was probably not a good constraint since, as mentioned above, it should be expected that more material would be needed in the composite case because of the low fluxes in the machine.

Thus, the following specifications were assumed:

- SMC and laminated cores to have the same total volume;

- the spider to be symmetrical with respect to the pole center line.

The rotor contained 8 permanent magnets and the stator had 12 salient poles. The magnet outer radius was set to $15.3 \mathrm{~mm}$, 


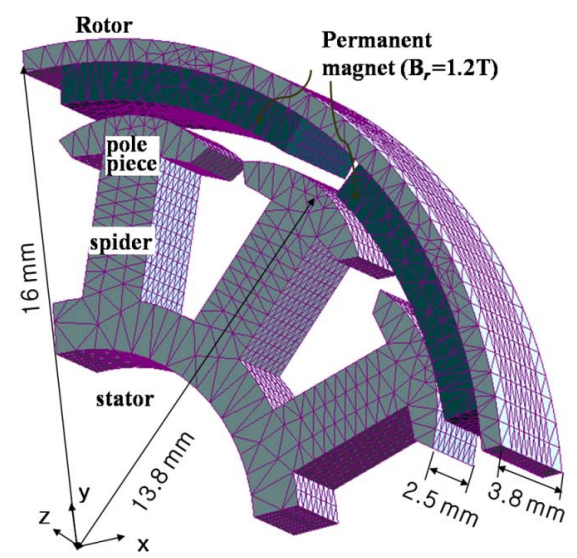

Fig. 3. One quarter model of a laminated spider stator.

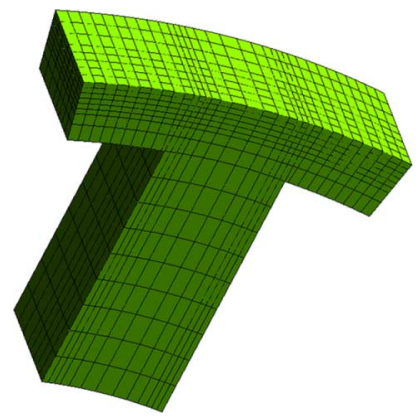

Fig. 4. Initial design domain.

the stator tooth depth to $2.5 \mathrm{~mm}$, and the magnet and yoke depth was $3.8 \mathrm{~mm}$.

The goal of the design was to optimize the stator pole shape to reduce the cogging torque and hence the objective function was specified as

$$
F=\sum\left(W_{i}^{\prime}-W_{o}^{\prime}\right)
$$

where $W_{i}^{\prime}$ is the co-energy at the $i$ th position and $W_{o}^{\prime}$ is a constant co-energy regardless of position. By minimizing (6), the energy variation becomes small at every rotor position and thus a minimum cogging torque profile is obtained.

A 3-D optimization was implemented based on CDSA and spline parameterization and the pole was set to be $2.5 \mathrm{~mm}$ long.

The initial design domain is shown in Fig. 4 and consists of 8 layers of design "cells," each having 381 cells for a total of 3048 cells per pole. The initial volume of the domain is set to be $58.4 \%$ greater than the optimal volume for the laminated steel solution.

The shape and topology optimization processes were implemented in MagNet [8].

The results for the laminated-core case are given in Fig. 5 demonstrating the improvement in cogging torque after shape optimization has been applied.

When topology optimization is applied using the SMC property data from Fig. 2, then the resulting structure is given in Fig. 6 and the performance in Fig. 7. Two issues arise from Fig. 7. The first is that the cogging torque actually increases during the optimization. The reason for this is the imposition of the volume constraint on the material. The process was started with a volume of material bigger than in the laminated version

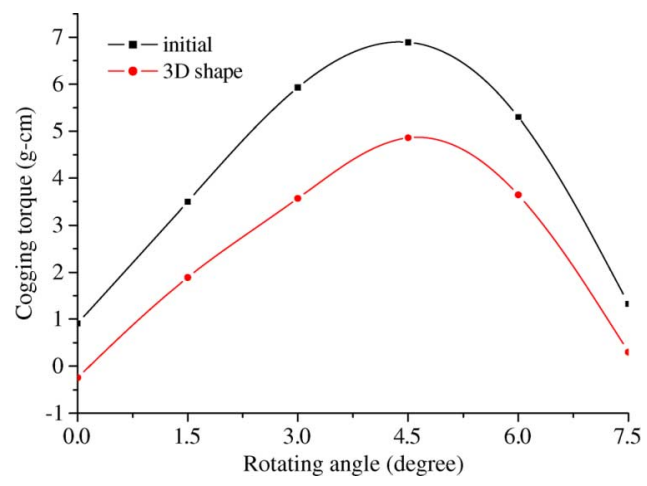

Fig. 5. Reduction in cogging torque after optimization for the motor with a laminated core.

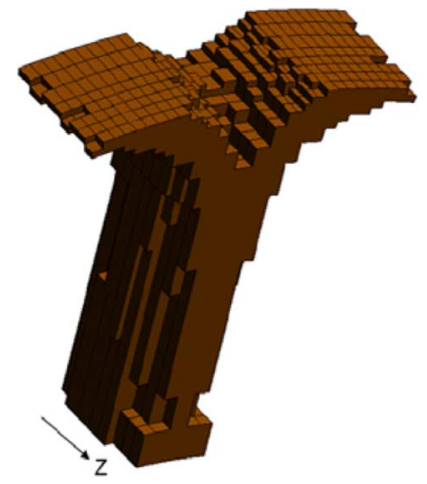

Fig. 6. Topology with SMC after 16 iterations.

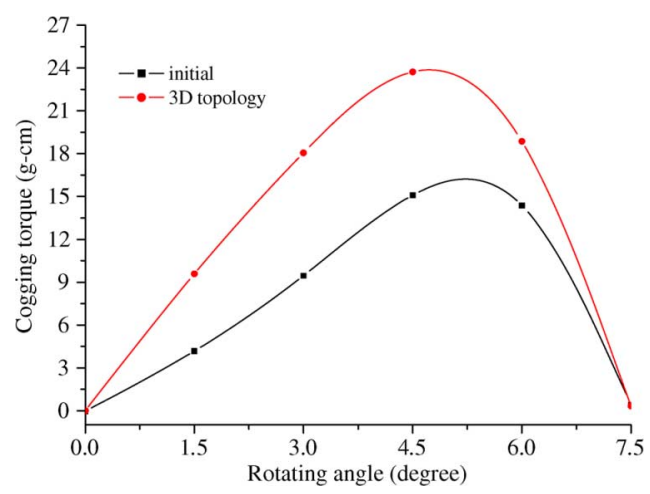

Fig. 7. Cogging torque performance for SMC pole.

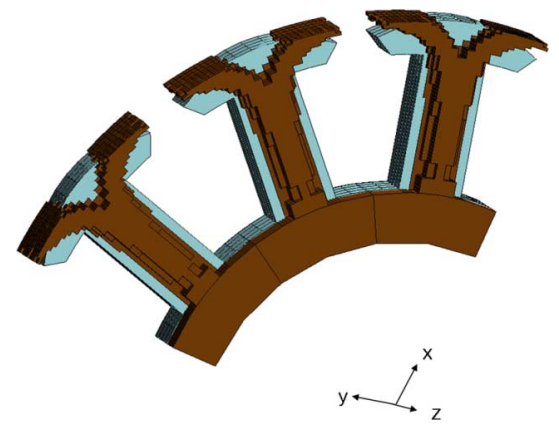

Fig. 8. Topolgy comparison between laminated and SMC cores.

and, as the volume is reduced, the fluxes increase leading to a higher torque. The second issue is that the cogging torque is about 5 times greater than for the laminated equivalent. One possible cause for this is that the optimization process has attempted to reduce the airgap of the motor in order to increase the fluxes. 


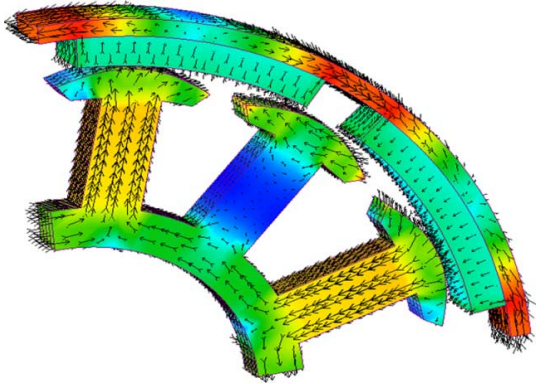

Fig. 9. Flux plot for laminated core-maximum B in stator is around $2.1 \mathrm{~T}$.

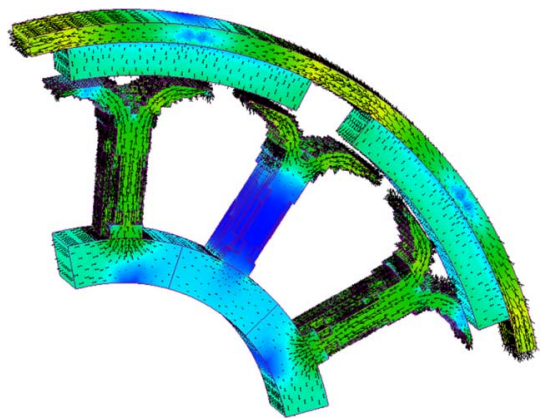

Fig. 10. Flux plot for SMC core-maximum B in stator is around $1.3 \mathrm{~T}$.

The shape difference between the two cores is shown in Fig. 8. Note that the SMC core (the darker of the two) has a very different structure from the lamination, particularly in the tips of the teeth. The field distributions in the two cores are shown in Figs. 9 and 10. Since cogging torque occurs because of the edges of the poles and magnets, it might be argued that the cell-type geometric structure that has resulted from the topology optimization artificially causes an increase in the cogging torque. However, an experiment depicted by Fig. 11 was attempted in which the cell-based topology structure was smoothed out by using a spline-curve function in SolidWorks [9] and the cogging torque recomputed based on a smooth geometry. The results showed no significant difference.

The shape created by the topology optimization is interesting in that the "notch" cut out of the pole might have been expected to add to the cogging torque and thus should not have been there; however, the volume constraint is possibly responsible for forcing the optimization process to follow a different route to the one predicted intuitively. It is probable that, if the laminated core had been designed using topology rather than shape optimization, it would also have received a notch as the material in the center of the pole face does not seem to be carrying a large amount of field.

While the test case above has failed to produce a "better" machine in electromagnetic sense, it has nevertheless achieved the main purpose of this paper to demonstrate the special features of topology and shape optimization of devices built from composite materials and has highlighted the unique design challenges.

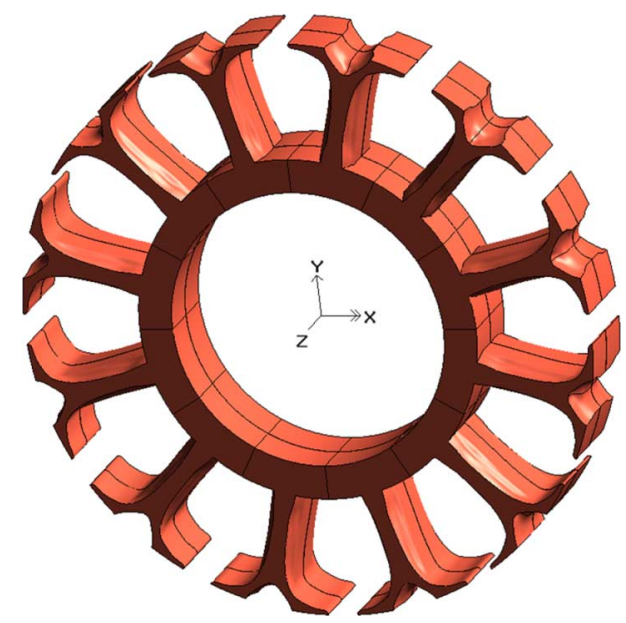

Fig. 11. Smoothing out the cell-based topology structure.

\section{CONCLUSION}

This paper has discussed the issues related to the topological design of electromagnetic devices using composite materials. By using a CDSA approach, the possible topologies can be explored almost automatically. It has been demonstrated how more design flexibility is available to benefit from the special features of composite materials.

\section{ACKNOWLEDGMENT}

This work was supported by KESRI (R-2005-7-068), which is funded by Ministry of Commerce, Industry and Energy (MOCIE), Korea.

\section{REFERENCES}

[1] J. Cea et al., "The shape and topological optimizations connection," ELSEVIER Comput. Methods Appl. Mech. Eng., vol. 118, pp. 713-726, 2000.

[2] S. Garreau et al., "The topological asymptotic for PDE systems: The elasticity case," SIAM J. Control Optim., vol. 39, pp. 1756-1778, 2001.

[3] D. N. Dyck et al., "A method of computing the sensitivity of electromagnetic quantities to changes in material and sources," IEEE Trans. Magn., vol. 30, no. 5, pp. 3415-3418, Sep. 1994.

[4] J.-K. Byun et al., "Topology optimization of electrical devices using mutual energy and sensitivity," IEEE Trans. Magn., vol. 35, no. 5, pp. 3718-3720, Sep. 1999.

[5] J.-H. Lee et al., "Faster calculation of sensitivity in the source current distribution problem using reciprocity theorem," IEEE Trans. Magn., vol. 37, no. 5, pp. 3596-3599, Sep. 2001.

[6] D.-H. Kim et al., "Applying continuum design sensitivity analysis combined with standard EM software to shape optimization in magnetostatic problems," IEEE Trans. Magn., vol. 40, no. 2, pp. 1156-1159, Mar. 2004

[7] S. Clenet, "Private Communication," Univ. Lille, Lille, France, Apr. 2008.

[8] “MagNet's User's Manual," Infolytica Corp., Montreal, QC, Canada, 2008.

[9] “SolidWorks User's Manual,” SolidWorks, Concord, MA, 2008. 